\title{
Genome sequencing and genomic characterization of a tigecycline-resistant Klebsiella pneumoniae strain isolated from the bile samples of a cholangiocarcinoma patient
}

Beiwen Zheng ${ }^{1,2 \dagger}$, Ang $\mathrm{Li}^{1,2 \dagger}$, Xiawei Jiang ${ }^{1,2}$, Xinjun Hu ${ }^{1,2}$, Jian Yao ${ }^{1,2}$, Lina Zhao ${ }^{1,2}$, Jinru Ji ${ }^{1,2}$, Min Ye $e^{1,2}$, Yonghong Xiao ${ }^{1,2^{*}}$ and Lanjuan $\mathrm{Li}^{1,2^{*}}$

\begin{abstract}
Background: The relationship between Klebsiella pneumoniae and nosocomial and community-acquired infections is well known, and K. pneumoniae resistance to most antibiotics is increasing worldwide. In contrast, tigecycline remains active against many bacterial strains, and serves as a last resort for treating multi-drug resistant bacterial infections. That tigecycline nonsusceptibility among K. pneumoniae isolates has been reported worldwide is worrying. However, the mechanisms of tigecycline resistance in $K$. pneumoniae are less well known. We report the genome sequence and genomic characterization of tigecycline-resistant K. pneumoniae strain 5422 isolated from the bile samples of a patient with cholangiocarcinoma.

Results: We sequenced the $K$. pneumoniae strain 5422 genome using next-generation sequencing technologies. Sequence data assembly revealed a 5,432,440-bp draft genome and $57.1 \%$ G + C content, which contained 5397 coding sequences. The genome has extensive similarity to other sequenced $K$. pneumoniae genomes, but also has several resistance-nodulation-cell division (RND) efflux pump genes that may be related to tigecycline resistance.

Conclusions: $K$. pneumoniae strain 5422 is resistant to multiple antibiotics. The genome sequence of the isolate and comparative analysis with other $K$. pneumoniae strains presented in this paper are important for better understanding of $K$. pneumoniae multi-drug resistance. The RND efflux pump genes identified in the genome indicate the presence of an antibiotic resistance mechanism prior to antibiotics overuse. The availability of the genome sequence forms the basis for further comparative analyses and studies addressing the evolution of the K. pneumoniae drug resistance mechanism and the K. pneumoniae transcriptome.
\end{abstract}

Keywords: Tigecycline-resistant, Klebsiella pneumoniae, Cholangiocarcinoma, Next-generation sequencing, Comparative genomics

\section{Background}

Klebsiella pneumoniae is a Gram-negative opportunistic pathogen from the family Enterobacteriaceae. The increasing resistance detected in clinical isolates has become a matter of significant concern, and K. pneumoniae is an ESKAPE (Enterococcus faecium, Staphylococcus aureus,

\footnotetext{
*Correspondence: xiao-yonghong@163.com; lij@zju.edu.cn

${ }^{\dagger}$ Equal contributors

'State Key Laboratory for Diagnosis and Treatment of Infectious Diseases, The First Affiliated Hospital, School of Medicine, Zhejiang University, Hangzhou 310003, China ${ }^{2}$ Collaborative Innovation Center for Diagnosis and Treatment of Infectious Diseases, Zhejiang University, Hangzhou 310003, China
}

K. pneumoniae, Acinetobacter baumannii, Pseudomonas aeruginosa, and Enterobacter species) pathogen [1,2]. Infections caused by $K$. pneumoniae have been identified worldwide. It is worth noting that it is a major cause of nosocomial and community-acquired infections [3,4]. Moreover, a distinct invasive syndrome that causes liver abscesses has been detected in increasing numbers in Asia in the past two decades, and this syndrome is emerging as a global disease [5,6]. Furthermore, $K$. pneumoniae harboring extended-spectrum $\beta$-lactamases, and more recently, carbapenemase, which confers resistance to 
multiple antibiotics, has become a significant clinical concern worldwide $[7,8]$. Mortality among patients infected with extremely resistant $K$. pneumoniae is high, perhaps due to the limited therapeutic options remaining [9].

Tigecycline, a novel class of glycylcyclines, is a minocycline derivative synthesized to overcome the major tetracycline resistance mechanisms and to extend its spectrum of activity to multidrug-resistant (MDR) bacteria [10]. Tigecycline has enhanced antimicrobial activity compared to tetracycline, and can overcome efflux pump systems and ribosome protection mechanisms, retaining activity against a broad range of both Gram-positive and Gram-negative bacteria [11-13]. K. pneumoniae resistance to most antibiotics is increasing globally. Nevertheless, tigecycline remains active against many bacterial strains, and serves as last resort for treating MDR bacterial infections [14]. However, tigecycline resistance has emerged recently and been widely reported in Enterobacteriaceae isolates. It is worth noting that tigecycline non-susceptibility among $K$. pneumoniae isolates has been reported from different continents and ranges between $0 \%$ and $50 \%$ [15-17].

Previous studies have suggested that tigecycline resistance in Enterobacteriaceae is attributed to resistancenodulation-cell division (RND)-type efflux pumps and transcriptional regulators of the efflux pump systems [15]. However, reports on the tigecycline resistance mechanisms in K. pneumoniae are rare. We hypothesized that a wide range of genes is involved in tigecycline resistance and contribute to decreased tigecycline susceptibility in K. pneumoniae, and performed whole-genome sequencing (WGS) to investigate this. We report the genome sequence of the tigecycline-resistant $K$. pneumoniae strain 5422 isolated from the bile samples of a patient with cholangiocarcinoma.

\section{Methods}

Strain information and growth conditions

Previously, we isolated strain 5422 from a bile sample obtained from a 54-year-old woman with bile duct cancer on February 25, 2012. After enrichment in Mueller-Hinton broth, the strain was identified as K. pneumoniae following the combination of its 16S rRNA gene sequencing and biochemical reaction results (VITEK 2 compact, bioMérieux, France). The strain exhibited high resistance to ciprofloxacin, cefotaxime, cefoxitin, ampicillin/sulbactam, sulfamethoxazole, tigecycline, tetracycline, and piperacillin, and was susceptible to imipenem, meropenem, and gentamicin. Multi-locus sequence typing revealed that it belonged to ST37 (unpublished data). The strain we reported here is available in the State Key Laboratory for Diagonosis and Treatment of Infectious Diseases, Zhejiang University.

\section{Genomic DNA extraction}

Late log-phase cells were harvested and lysed with EDTA, lysozyme, and detergent treatment, followed by proteinase $\mathrm{K}$ and RNase digestion. Genomic DNA was extracted using a DNeasy Blood \& Tissue Kit (Qiagen, Germany) according to the manufacturer's recommended protocol. Genomic DNA yield, purity, and concentration was evaluated using $0.7 \%$ agarose gel electrophoresis with $\lambda$-Hind III digest DNA Marker and measured using a NanoDrop 1000 Spectrophotometer (Thermo Fisher Scientific, USA). The genomic DNA was stored at $-20^{\circ} \mathrm{C}$.

\section{Genome sequencing and annotation}

Whole-genome shotgun sequencing of $K$. pneumoniae strain 5422 was performed using a standard run of IlluminaHiSeq2000 sequencing by generating paired-end libraries (500-bp insert size) with a $2 \times 100$ pair-end sequencing strategy according to the manufacturer's instructions. Clean reads were assembled into scaffolds using Velvet version 1.2.07 [18], and then we used PAGIT (PostAssembly Genome Improvement Toolkit) [19] to extend the initial contiguous sequences (contigs) and correct sequencing errors. We identified tRNAs and rRNAs using tRNAscan-SE [20] and RNAmmer [21], respectively. Open reading frames (ORFs) were identified using Glimmer version 3.0 [22]. The genome was annotated using the RAST (Rapid Annotation using Subsystem Technology) server [23]. The classification of some predicted genes and pathways was analyzed using the COGs (Clusters of Orthologous Groups of proteins) [24] and KEGG (Kyoto Encyclopedia of Genes and Genomes) [25] databases. Stretches of amino acids containing the efflux pump genes were searched using BLAST (Basic Local Alignment Search Tool, http://blast.ncbi.nlm.nih.gov/Blast.cgi); protein-coding sequences were further BLAST-searched against the Antibiotic Resistance Database (ARDB) [26]. To find genes with hypothetical or putative functions, we aligned genes against the National Center for Biotechnology Information (NCBI) nucleotide sequence database (downloaded September 20, 2013) using NCBI BLASTn: we accepted only hits with identity of $\geq 0.95$, coverage $\geq 0.9$, and putative or hypothetical reference gene annotation.

\section{Initial comparative genomic and phylogenetic analysis}

For comparative analysis, we downloaded the reference genome sequences of the closest genetic relatives of $K$. pneumoniae strain 5422 and representative strains from the NCBI website: K. pneumoniae LCT-KP182 (ATRN 00000000), K. pneumoniae LCT-KP289 (ATRO00000000), K. pneumoniae subsp. pneumoniae LZ (AJVY00000000), K. pneumoniae ATCC BAA-2146 (AOCV00000000), K. pneumoniae 123578 (PRJNA199972), K. pneumoniae NB60 (AZAP00000000), K. pneumoniae subsp. pneumoniae 1084 (СР003785), K. pneumoniae subsp. pneumoniae 
A

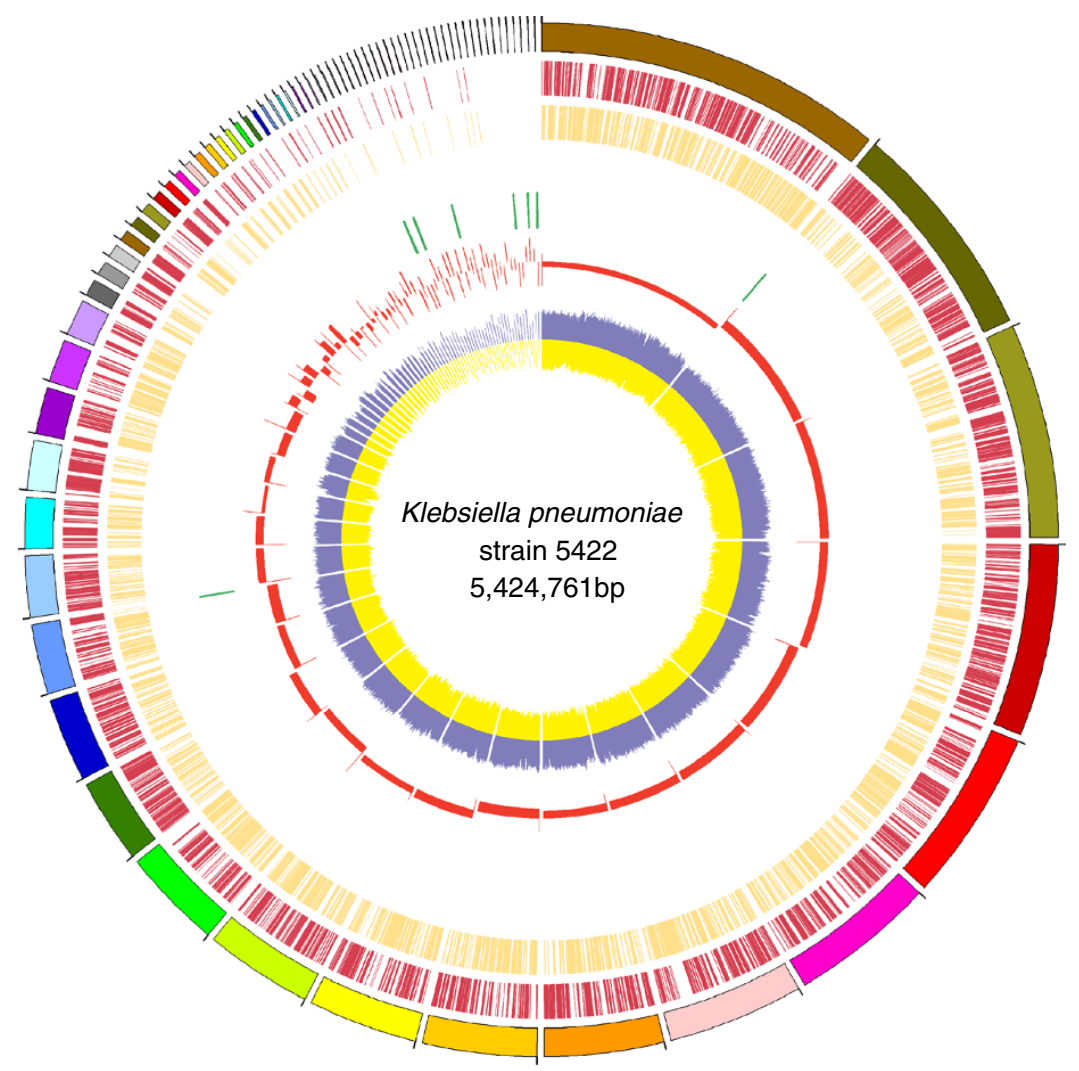

B

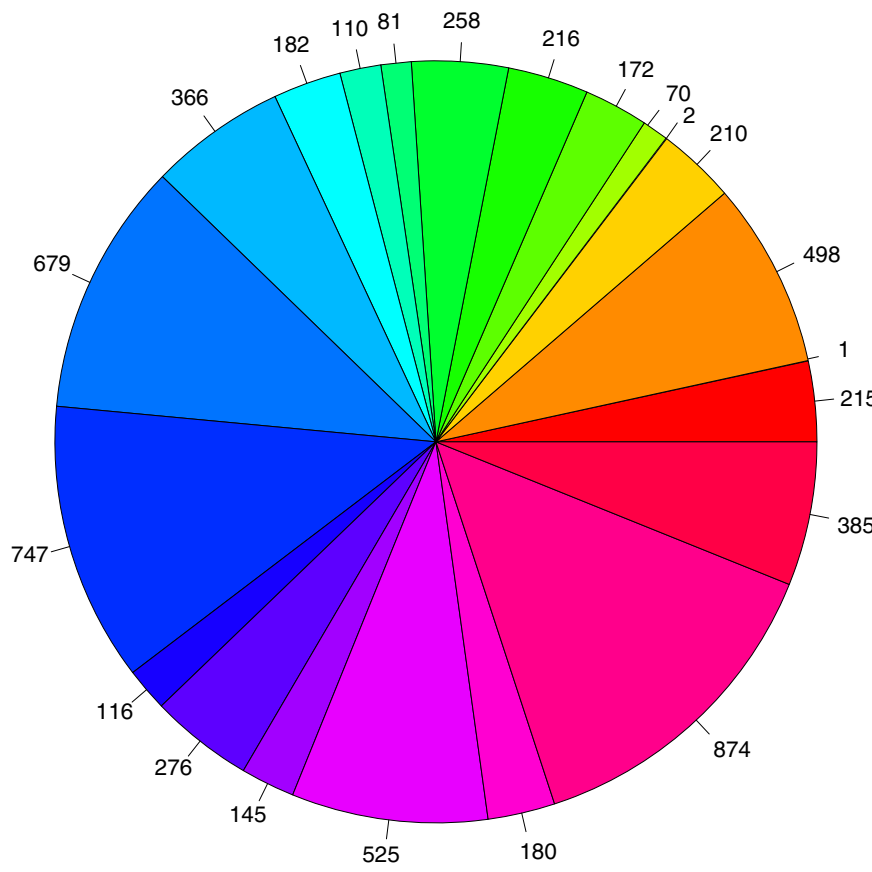

- Translation, ribosomal structure and biogenesis (215) - RNA processing and modification (1)

- Transcription (498)

- Replication, recombination and repair (210)

Chromatin structure and dynamics (2)

- Cell cycle control, cell division (70)

- Defense mechanisms (172)

- Signal transduction mechanisms (216)

I Cell wall/membrane/envelope biogenesis (258)

- Cell motility (81)

- Intracellular traffickingrt (110)

1. Posttranslational modification (182)

- Energy production and conversion (366)

- Carbohydrate transport and metabolism (679)

- Amino acid transport and metabolism (747)

- Nucleotide transport and metabolism (116)

- Coenzyme transport and metabolism (276)

- Lipid transport and metabolism (145)

- Inorganic ion transport and metabolism (525)

- Secondary metabolites biosynthesis (180)

- General function prediction only (874)

- Function unknown (385)

Figure 1 (See legend on next page.) 
(See figure on previous page.)

Figure 1 Genome map and phylogenetic analysis. A. Circular map of the genome generated using Circos. Circles from outside to inside: 1 , contigs were arrange in clockwise direction from large to small; 2, CDS on forward strand; 3, CDS on reverse strand; 4, tRNA genes; 5, rRNA genes; 6, GC-skew (window size of $10 \mathrm{~kb}$ ); and 7, blue indicates C content and yellow indicates G content (step size 500 bp). B. COGs distribution of K. pneumoniae strain 5422 .

\section{A}

Subsystem Category Distribution

\section{Coverage}

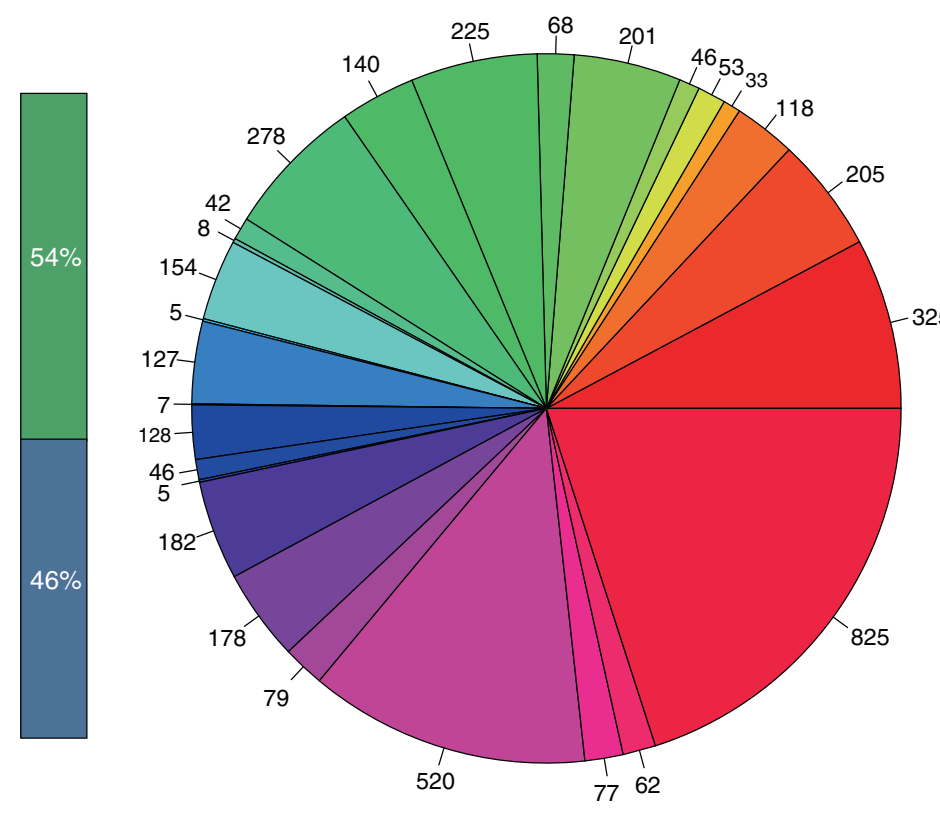

\section{Subsystem Feature}

- Cofactors, Vitamins, Prosthetic Groups, Pigments (325)

- Cell Wall and Capsule (205)

- Virulence, Disease and Defense (118)

- Potassium metabolism (33)

Miscellaneous (53)

- Phages, Prophages, Transposable elements, Plasmids (46)

- Membrane Transport (201)

- Iron acquisition and metabolism (68)

- RNA Metabolism (225)

- Nucleosides and Nucleotides (140)

- Protein Metabolism (278)

- Cell Division and Cell Cycle (42)

- Motility and Chemotaxis (8)

- Regulation and Cell signaling (154)

- Secondary Metabolism (5)

- DNA Metabolism (127)

- Regulons (7)

- Fatty Acids, Lipids, and Isoprenoids (128)

- Nitrogen Metabolism (46)

- Dormancy and Sporulation (5)

- Respiration (182)

- Stress Response (178)

- Metabolism of Aromatic Compounds (79)

- Amino Acids and Derivatives (520)

- Sulfur Metabolism (77)

- Phosphorus Metabolism (62)

- Carbohydrates (825)

B

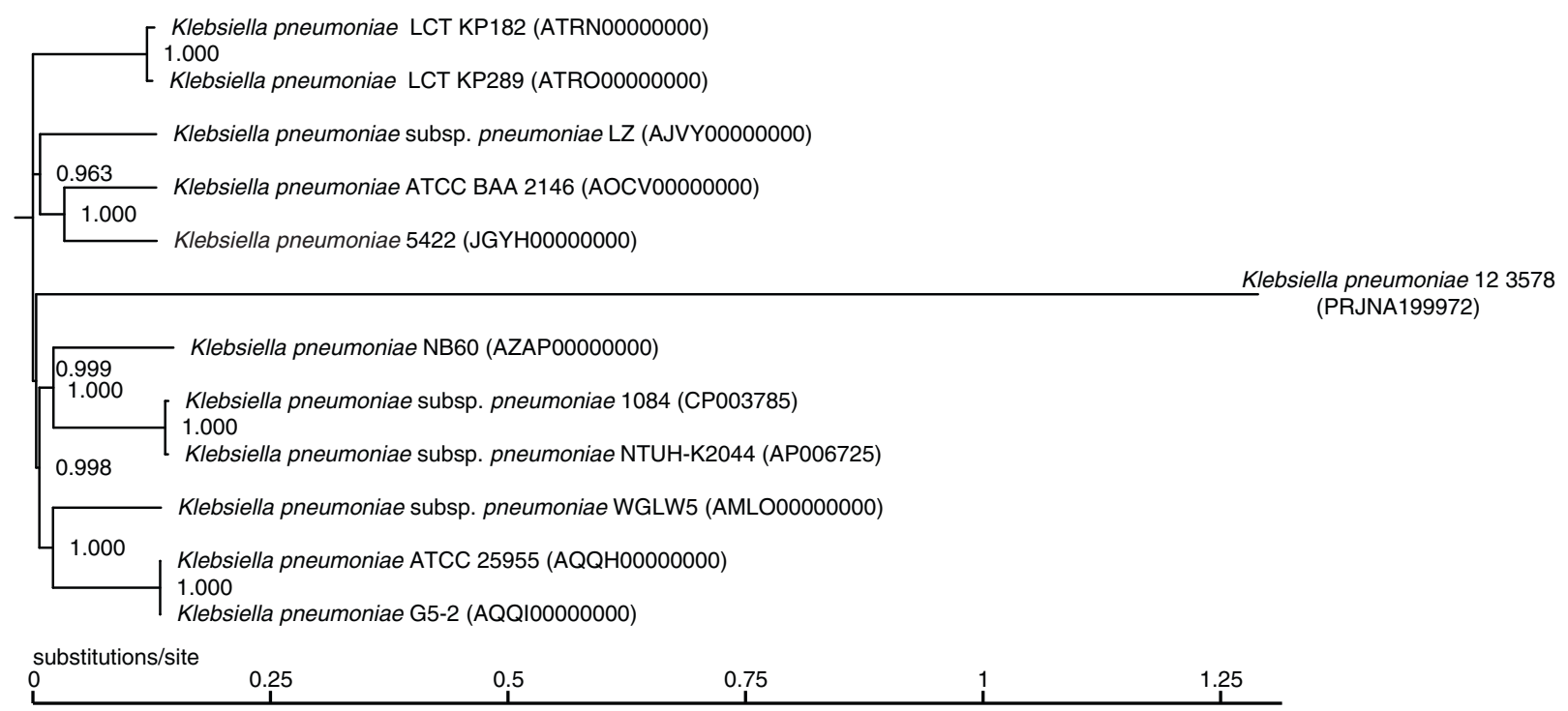

Figure 2 Subsystem distribution and phylogenetic analysis. A. Distribution of genes assigned to SEED subsystems (based on the RAST annotation server). B. Phylogenetic relationships (based on WGS and SNPS) of 12 K. pneumoniae strains and their genomic distance analysis. The snpTree server output used assembled genomes as input data. 
NTUH-K2044 (AP006725), K. pneumoniae subsp. pneumoniae WGLW5 (AMLO00000000), K. pneumoniae ATCC 25955 (AQQH00000000), and K. pneumoniae G5-2 (AQQI00000000). Whole-genome alignments, single-nucleotide polymorphism (SNP) identification, and phylogenetic tree construction were performed using snpTree version 1.1, a server for online automatic SNP analysis of assembled genomes (http://cge.cbs.dtu. $\mathrm{dk} /$ services/snpTree-1.1/) [27].

\section{Quality assurance}

The 16S rDNA gene from the draft genome was used to check for contamination. Further VITEK biochemical identification data confirmed that the strain 5422 belonged to $K$. pneumoniae. Bioinformatics assessment of potential contamination of the genomic library by allochthonous microorganisms was achieved using the BLAST non-redundant database.

\section{Initial findings}

\section{Genome characteristics and phylogenetic analysis}

Filtered 520.8 M clean reads were assembled into scaffolds, and corresponding 99-fold coverage of the genome was generated. The draft genome sequence of $K$. pneumoniae strain 5422 was $5,432,440$ bp in size and had a $\mathrm{G}+\mathrm{C}$ content of $57.1 \%$ in 133 contigs, with N50 spanning 105,586 bp. Figure 1A depicts the overall genome profile.
Annotation of this assembly identified 5,397 coding sequences (CDSs), 65 tRNAs (excluding 0 pseudo tRNAs), and incomplete rRNA operons (three small subunit rRNAs, four large subunit rRNAs). We assigned putative function or hypothetical proteins to 1,478 protein-coding genes. We categorized 4,218 genes into COGs functional groups (including putative or hypothetical genes, Figure 1B). For COGs distribution, $\mathrm{R}$ (general function prediction only; 874 ORFs), E (amino acid transport and metabolism; 747 ORFs), G (carbohydrate metabolism and transport; 679 ORFs), and P (inorganic ion transport and metabolism; 525 ORFs) were abundant categories ( $>10 \%$ of total COGs matched counts).

Figure $2 \mathrm{~A}$ illustrates the subsystem distribution and general information on the potential functional distribution of K. pneumoniae strain 5422. Genes responsible for carbohydrates (825 ORFs); amino acids and derivatives (520 ORFs); and cofactors, vitamins, prosthetic groups, and pigments (325 ORFs) were abundant among the SEED subsystem categories. Based on the raw reads and assembled genomes from published $K$. pneumoniae WGS data sets, we conducted phylogenetic analysis on tree topology and the SNP positions of the reference genome to identify the most closely related organism. The phylogenetic tree based on whole-genome SNPs showed that the closest ancestor to K. pneumoniae strain 5422 was K. pneumoniae ATCC BAA-2146 (Figure 2B),

Table 1 Summary of CDSs annotated to RND efflux pump genes

\begin{tabular}{llllll}
\hline Aligned protein & Query sequence length & Coverage (\%) & Hit length & Identity (\%) & Description \\
\hline AcrA & 240 & 64.17 & 146 & 60.08 & Probable RND efflux membrane fusion protein \\
OqxB & 217 & 141 & 64.98 & $\begin{array}{l}\text { Probable RND efflux system inner membrane } \\
\text { transporter CmeB }\end{array}$ \\
OqxB & 181 & 18.53 & 110 & 60.77 & Probable RND multi-drug efflux transporter \\
OqxB & 160 & 15.27 & 105 & 65.62 & Probable RND efflux system inner membrane \\
transporter CmeB
\end{tabular}


which is the first US isolate found to encode New Delhi metallo- $\beta$-lactamase 1 (NDM-1), eight $\beta$-lactamases, and 15 additional antibiotic-resistance enzymes [28,29].

\section{RND efflux pumps in K. pneumoniae strain 5422}

The RND family members are important mediators of MDR in Gram-negative bacteria. The AcrAB-TolC system in Escherichia coli and the MexAB-OprM complexes in $P$. aeruginosa are extremely well characterized, and the three-dimensional structures of various components have been resolved [30]. In the genome of K. pneumoniae strain 5422, 16 genes were indicated as probable efflux pumps or translational regulators based on their sequence similarity to known RND efflux pump genes (Table 1).

\section{Future directions}

The rapid progress of WGS has permitted detailed investigation of genetic differences between bacterial isolates with different phenotypic characteristics. Wholegenome studies of $K$. pneumoniae have mainly focused on comparing either distinct antibiotic-susceptible and MDR strains or related isolates from different patients. Therefore, large-scale genomic sequencing and comparative genome analysis of tigecycline-resistant, tigecycline non-susceptible, and tigecycline-susceptible clinical isolates will identify the differences in the genomic content of this species and yield evolutionary information on the development of tigecycline resistance through mutations. Moreover, further studies involving extensive high-throughput mRNA sequencing (RNA-Seq) experiments to significantly improve annotation and to provide exceptionally robust analysis of RNA expression under selective antibiotic pressure are warranted.

\section{Ethics approval}

This research was approved by the Research Ethics Committee of the First Affiliated Hospital, School of Medicine, Zhejiang University, and informed consent was obtained from the patient.

\section{Availability of supporting data}

This Whole Genome Shotgun project has been deposited at DDBJ/EMBL/GenBank under the accession JGYH00000000. The version described in this paper is version JGYH01000000.

\section{Competing interests}

The authors have no competing interests.

\section{Authors' contributions}

BWZ, XJH, LNZ, JRJ and MY performed microbiology and molecular biology experiments. XWJ and JY generated and analyzed the sequencing data. BWZ and AL participated all discussions of data analysis and write the manuscript. $B W Z, Y H X$ and $L L$ were involved in overall experimental design. All authors read and approved the final manuscript.

\section{Acknowledgments}

This work was supported by the National Natural Science Foundation of China (Grant No. 81361138021 and 81301461), Zhejiang Provincial Natural Science Foundation of China (Grant No. LQ13H190002) and the Scientific Research

Foundation of Zhejiang Provincial Health Bureau (Grant No. 2012KYB083).

Received: 11 July 2014 Accepted: 2 September 2014

Published online: 27 September 2014

\section{References}

1. Boucher HW, Talbot GH, Bradley JS, Edwards JE, Gilbert D, Rice LB, Scheld M, Spellberg B, Bartlett J: Bad bugs, no drugs: no ESKAPE! An update from the Infectious Diseases Society of America. Clin Infect Dis 2009, 48:1-12.

2. Rice LB: Federal funding for the study of antimicrobial resistance in nosocomial pathogens: no ESKAPE. J Infect Dis 2008, 197:1079-1081.

3. Moore R, O'Shea D, Geoghegan T, Mallon PW, Sheehan G: Communityacquired Klebsiella pneumoniae liver abscess: an emerging infection in Ireland and Europe. Infection 2013, 41:681-686.

4. Henderson KL, Muller-Pebody B, Johnson AP, Wade A, Sharland M, Gilbert R: Community-acquired, healthcare-associated and hospital-acquired bloodstream infection definitions in children: a systematic review demonstrating inconsistent criteria. J Hosp Infect 2013, 85:94-105.

5. Siu LK, Yeh KM, Lin JC, Fung CP, Chang FY: Klebsiella pneumoniae liver abscess: a new invasive syndrome. Lancet Infect Dis 2012, 12:881-887.

6. Lin JC, Koh TH, Lee N, Fung CP, Chang FY, Tsai YK, Ip M, Siu LK: Genotypes and virulence in serotype K2 Klebsiella pneumoniae from liver abscess and non-infectious carriers in Hong Kong, Singapore and Taiwan. Gut Pathog 2014, 6:21.

7. Nordmann P, Cuzon G, Naas T: The real threat of Klebsiella pneumoniae carbapenemase-producing bacteria. Lancet Infect Dis 2009, 9:228-236.

8. Richter SN, Frasson I, Franchin E, Bergo C, Lavezzo E, Barzon L, Cavallaro A, Palu G: KPC-mediated resistance in Klebsiella pneumoniae in two hospitals in Padua, Italy, June 2009-December 2011: massive spreading of a KPC-3-encoding plasmid and involvement of non-intensive care units. Gut Pathog 2012, 4:7.

9. Munoz-Price LS, Poirel L, Bonomo RA, Schwaber MJ, Daikos GL, Cormican M, Cornaglia G, Garau J, Gniadkowski M, Hayden MK, Kumarasamy K, Livermore DM, Maya JJ, Nordmann P, Patel JB, Paterson DL, Pitout J, Vilegas MV, Wang H, Woodford N, Quinn JP: Clinical epidemiology of the global expansion of Klebsiella pneumoniae carbapenemases. Lancet Infect Dis 2013, 13:785-796.

10. Petersen PJ, Jacobus NV, Weiss WJ, Sum PE, Testa RT: In vitro and in vivo antibacterial activities of a novel glycylcycline, the 9-t-butylglycylamido derivative of minocycline (GAR-936). Antimicrob Agents Chemother 1999, 43:738-744.

11. Jenner L, Starosta AL, Terry DS, Mikolajka A, Filonava L, Yusupov M Blanchard SC, Wilson DN, Yusupova G: Structural basis for potent inhibitory activity of the antibiotic tigecycline during protein synthesis. Proc Natl Acad Sci U S A 2013, 110:3812-3816.

12. Brust $K$, Evans A, Plemmons R: Tigecycline in treatment of multidrugresistant Gram-negative bacillus urinary tract infections: a systematic review. J Antimicrob Chemother 2014.

13. Kanj SS, Whitelaw A, Dowzicky MJ: In vitro activity of tigecycline and comparators against Gram-positive and Gram-negative isolates collected from the Middle East and Africa between 2004 and 2011. Int J Antimicrob Agents 2014, 43:170-178.

14. Lin YT, Wang FD, Chan YJ, Fu YC, Fung CP: Clinical and microbiological characteristics of tigecycline non-susceptible Klebsiella pneumoniae bacteremia in Taiwan. BMC Infect Dis 2014, 14:1.

15. Sun Y, Cai Y, Liu X, Bai N, Liang B, Wang R: The emergence of clinical resistance to tigecycline. Int J Antimicrob Agents 2013, 41:110-116.

16. Nigo M, Cevallos CS, Woods K, Flores VM, Francis G, Perlman DC, Revuelta M, Mildvan D, Waldron M, Gomez T, Koshy S, Jodlowski T, Riley W, Ruhe JJ: Nested case-control study of the emergence of tigecycline resistance in multidrug-resistant Klebsiella pneumoniae. Antimicrob Agents Chemother 2013, 57:5743-5746.

17. Huang TD, Berhin C, Bogaerts P, Glupczynski Y: In vitro susceptibility of multidrug-resistant Enterobacteriaceae clinical isolates to tigecycline. J Antimicrob Chemother 2012, 67:2696-2699.

18. Zerbino DR, Birney E: Velvet: algorithms for de novo short read assembly using de Bruijn graphs. Genome Res 2008, 18:821-829. 
19. Swain MT, Tsai IJ, Assefa SA, Newbold C, Berriman M, Otto TD: A Post-Assembly Genome-Improvement Toolkit (PAGIT) to obtain annotated genomes from contigs. Nat Protoc 2012, 7:1260-1284.

20. Lowe TM, Eddy SR: tRNAscan-SE: a program for improved detection of transfer RNA genes in genomic sequence. Nucleic Acids Res 1997, 25:0955-0964.

21. Lagesen $K$, Hallin P, Rødland EA, Stærfeldt H-H, Rognes T, Ussery DW: RNAmmer: consistent and rapid annotation of ribosomal RNA genes. Nucleic Acids Res 2007, 35:3100-3108.

22. Delcher AL, Bratke KA, Powers EC, Salzberg SL: Identifying bacterial genes and endosymbiont DNA with Glimmer. Bioinformatics 2007, 23:673-679.

23. Aziz RK, Bartels D, Best AA, De Jongh M, Disz T, Edwards RA, Formsma K, Gerdes S, Glass EM, Kubal M, Meyer F, Olsen GJ, Olson R, Osterman AL, Overbeek RA, McNeil LK, Paarmann D, Paczian T, Parrello B, Pusch GD, Reich C, Stevens R, Vassieva O, Vonstein V, Wilke A, Zagnitko O: The RAST Server: rapid annotations using subsystems technology. BMC Genomics 2008, 9:75.

24. Tatusov RL, Fedorova ND, Jackson JD, Jacobs AR, Kiryutin B, Koonin EV Krylov DM, Mazumder R, Mekhedov SL, Nikolskaya AN, Rao BS, Smirnov S, Sverdlov AV, Vasudevan S, Wolf YI, Yin JJ, Natale DA: The COG database: an updated version includes eukaryotes. BMC Bioinformatics 2003, 4:41

25. Mitra S, Rupek P, Richter DC, Urich T, Gilbert JA, Meyer F, Wilke A, Huson $\mathrm{DH}$ : Functional analysis of metagenomes and metatranscriptomes using SEED and KEGG. BMC Bioinformatics 2011, 12 Suppl 1:S21.

26. Liu B, Pop M: ARDB-Antibiotic Resistance Genes Database. Nucleic Acids Res 2009, 37:D443-D447.

27. Leekitcharoenphon P, Kaas RS, Thomsen MC, Friis C, Rasmussen S, Aarestrup FM: snpTree-a web-server to identify and construct SNP trees from whole genome sequence data. BMC Genomics 2012, 13 Suppl 7:S6.

28. Broberg $C A$, Palacios M, Miller $\mathrm{VL}$ : Whole-genome draft sequences of three multidrug-resistant klebsiella pneumoniae strains available from the American type culture collection. Genome Announc 2013, 1:e00312-e00313.

29. Hudson CM, Bent ZW, Meagher RJ, Williams KP: Resistance determinants and mobile genetic elements of an NDM-1-encoding Klebsiella pneumoniae strain. PLoS One 2014, 9:e99209.

30. Alvarez-Ortega C, Olivares J, Martinez JL: RND multidrug efflux pumps: What are they good for? Front Microbiol 2013, 4:7.

doi:10.1186/s13099-014-0040-2

Cite this article as: Zheng et al:: Genome sequencing and genomic characterization of a tigecycline-resistant Klebsiella pneumoniae strain isolated from the bile samples of a cholangiocarcinoma patient. Gut Pathogens 2014 6:40

\section{Submit your next manuscript to BioMed Central and take full advantage of:}

- Convenient online submission

- Thorough peer review

- No space constraints or color figure charges

- Immediate publication on acceptance

- Inclusion in PubMed, CAS, Scopus and Google Scholar

- Research which is freely available for redistribution 